\title{
Aerial Survey and Scanning Point Cloud Data Acquisition \& Modeling Technology for Hydropower Engineering
}

\author{
Shangwei LIU $^{1,2}$, Ying WANG ${ }^{1}$, Hua JIANG ${ }^{1,3}$, Qun WEI ${ }^{1,3}$, Jun XIAO ${ }^{1, \text { a }}$ \\ ${ }^{1}$ University of Chinese Academy of Sciences, Beijing, 100049, China; \\ ${ }^{2}$ School of Water Conservancy, North China University of Water Resources and Electric Power, \\ Zhengzhou, 450045, China; \\ ${ }^{3}$ Steel Structure and Engineering Research Institute, North China University of Water Resources \\ and Electric Power, Zhengzhou, 450045, China; \\ aemail: xiaojun@ucas.ac.cn
}

Keywords: Aerial photogrammetry, Laser scanning, Point cloud data, Fitting processing, Reverse modeling

\begin{abstract}
Through a systematic research on the application of methods for acquiring point cloud data concerning aerial surveying and laser scanning of modern water conservancy projects, a kind of advanced aerial photogrammetry and laser point cloud scanning and acquiring technology is proposed and tools are developed for processing of point cloud data of modern water conservancy projects and reverse modeling technology. Combined with a specific hydropower project, detailed application is carried out step by step from the aspects of station distribution and acquisition of point cloud data, splicing and redundancy removal processing of data and establishment of 3D models and reverse 3D modeling is implemented, realizing the basic modeling for informationization of modern hydropower projects and developing a new method and thinking for surveying work of hydropower projects.
\end{abstract}

\section{Introduction}

In the early design stage of a water resources and hydropower engineering, normal mapping work still stays in the stage of using total station and GPS RTK field surveying and mapping, which are two backward ways of surveying and mapping. To change this situation, a research is made on the data acquisition technology for hydropower engineering in combination with the current advanced aerial survey and laser point cloud scanning technology, developing a new method and thinking for the surveying of hydropower engineering.

\section{Acquisition Method of Point Cloud Data}

\section{Aerial photogrammetry}

Aerial photogrammetry means the work to take pictures of the ground continuously with aero-photographic apparatus on the plane and to draw the topographical map in combination with such procedures as ground control point measurement, annotation and stereo surveying and mapping[1].

Md4-1000 type quadrotor unmanned aerial vehicle (see Fig. 1 (a)) is a globally leading small automatic driving and vertical take-off and landing unmanned aircraft system. By microwave image real-time transmission equipment and data acquisition equipment of the unmanned aircraft system, surface deformation information of the rock mass at rocky slopes can be acquired rapidly (see Fig. 1 (b)). 


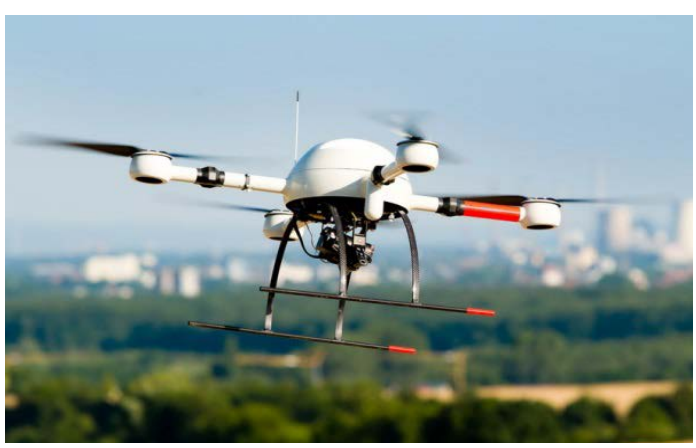

(a) MD4-1000 unmanned aerial vehicle

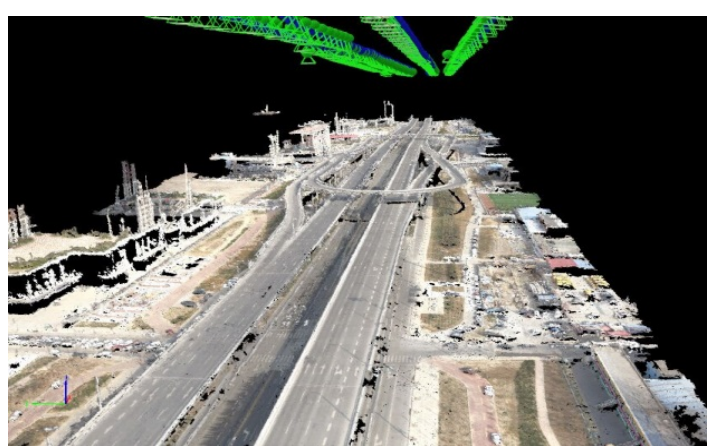

(b) Spatial 3D ray diagram

\section{D laser scanning technology}

Fig. 1 Aerial Photogrammetry

3D laser scanning technology is a high and new technology recently developed in the world. Data measured each time by existing 3D laser scanner not only include the information of points X, $\mathrm{Y}$ and $\mathrm{Z}$, but also include R, G and B color information as well as reflectivity of objects. 3D laser scanner has been successfully applied in such fields as protection of cultural relics, surveying of urban architectures, topographic surveying and mapping, mining, deformation monitoring, factories, large structures, etc[2] (see Fig. 2).

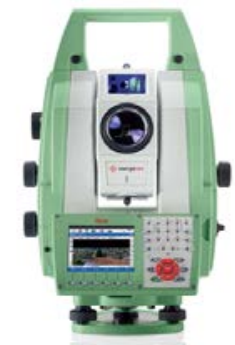

(a) Ms50 total station scanner

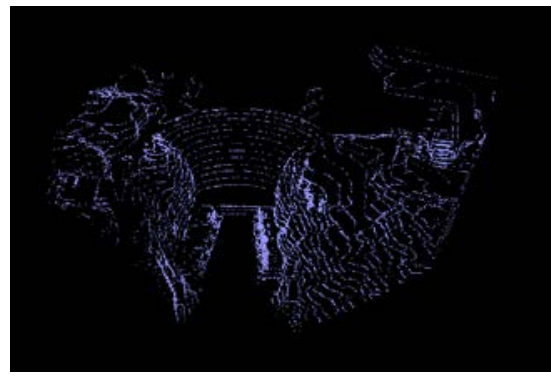

(b) Point cloud of a dam

\section{D structured light scanner}

Fig. 2 3D laser total station scanner

3D structured light scanner is a high-tech product integrating optical, mechanical, electrical and computer technology, mainly used to acquire 3D coordinates of the outer surface of objects and 3D digital models of objects (see Fig. 3). It stripe images are processed at a high speed through advanced and unique 3D image processing software to work out the accurate spatial coordinates of the corresponding point of each pixel $(X, Y, Z)$ and the vivid color data $(R, G, B)$, generate 3D (color) surface point cloud data and realize the optical 3D (color) digital scanning of almost all visible objects and human bodies [3].

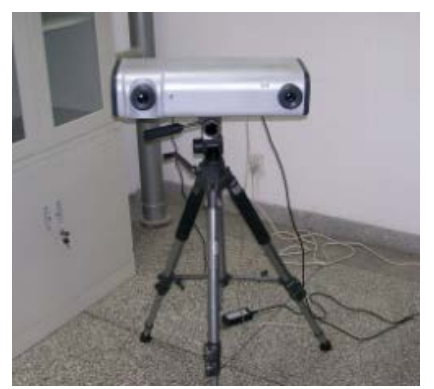

(a)CaMega structured light scanner

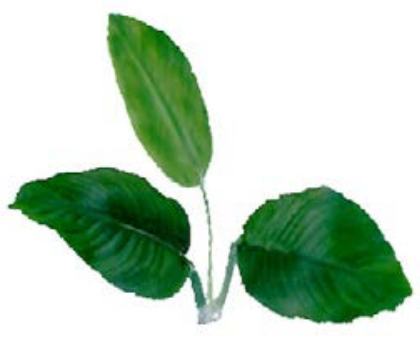

(b) Structured light scanner acquiring plant point cloud Fig. 3 Surveying with structured light scanner

\section{Portable 3D scanner}

SCAN 3D ${ }^{\mathrm{TM}}$ is a kind of portable 3D scanning equipment which can provide quick and reliable surveying and acquire full-color 3D data. It is a high-speed 3D laser scanner used for detailed surveying and digital archiving and needs only several minutes to provide very detailed 3D images of complex environment and geometrical shapes (see Fig. 4). 


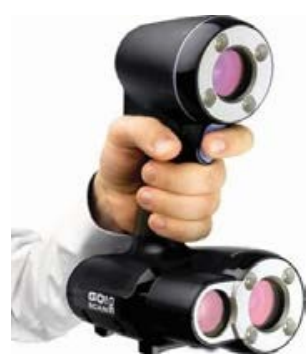

(a) SCAN 3D ${ }^{\mathrm{TM}} 3 \mathrm{D}$ scanner

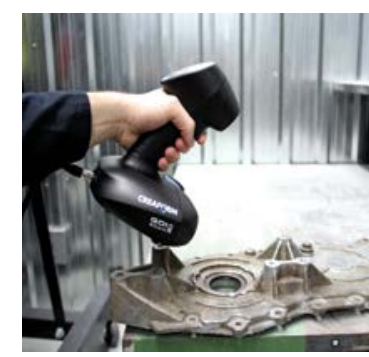

(b) Portable scanner acquiring point cloud

\section{Reverse Modeling Method of Point Cloud Data}

Point cloud information includes not only spatial coordinates of the point cloud data, but also color information (R, G, B) and brightness information (I) of the point cloud data; therefore, each cloud data point include seven items of information (X, Y, Z, R, G, B, I), establishing template database and transformation formats[5-9].

Processing of contour of fitting equation

All data we have now are the general equations of different planes, and unparallel planes must have a common line of intersection. Calculation method:

Firstly, take the possible value of any parameter of $\mathrm{x}, \mathrm{y}, \mathrm{z}$ on the common line of intersection, and then substitute it into equation set

$$
\left\{\begin{array}{l}
A_{1} x_{0}+B_{1} y_{0}+C_{1} z_{0}+D=0 \\
A_{2} x_{0}+B_{2} y_{0}+C_{2} z_{0}+D=0
\end{array}\right.
$$

Hence the common point ( $\mathrm{x} 0, \mathrm{y} 0, \mathrm{z} 0)$.

Next, calculate the direction vector of the straight line with the following method:

$$
\begin{array}{r}
s=n_{1} \times n_{2}=\left|\begin{array}{ccc}
i & j & k \\
A_{1} & B_{1} & C_{1} \\
A_{2} & B_{2} & C_{2}
\end{array}\right| \\
\text { Hence }\left\{\begin{array}{l}
m=B_{1} C_{2}-B_{2} C_{1} \\
n=A_{2} C_{1}-A_{1} C_{2} \\
p=A_{1} B_{2}-A_{2} B_{1}
\end{array}\right.
\end{array}
$$

Hence parameter equation of the straight line.

Equations of a spatial straight line include:

$$
\text { General equation: }\left\{\begin{array}{l}
A_{1} x+B_{1} y+C_{1} z+D=0 \\
A_{2} x+B_{2} y+C_{2} z+D=0
\end{array}\right.
$$

Symmetric equation:

$$
\frac{x-x_{0}}{m}=\frac{y-y_{0}}{n}=\frac{z-z_{0}}{p}
$$

Parameter equation:

$$
\left\{\begin{array}{l}
x=x_{0}+m t \\
y=y_{0}+n t \\
z=z_{0}+p t
\end{array}\right.
$$

Considering that it can't be guaranteed that all of $\mathrm{m}, \mathrm{n}$ and $\mathrm{p}$ are non-zero, we choose the parameter equation of spatial straight line in calculation.

Contour fitting processing will provide the most direct auxiliary line for reverse modeling. Select two unparallel planes from the plane list, and calculate their line of intersection to generate the line of intersection (ray, as shown in Fig. 5) of the two planes in Civil3D space. 


\section{Processing of center line of fitting equation}

Given a set of $\operatorname{data}\left(x_{i}, y_{i}\right), i=1,2, \cdots, m$, fit a straight line $p(x)=a+b x$, and the mean square error is

$$
Q(a, b)=\sum_{i=1}^{m}\left(p\left(x_{i}\right)-y_{i}\right)^{2}=\sum_{i=1}^{m}\left(a+b x_{i}-y_{i}\right)^{2}
$$

In the theory of calculus, the minimum of $Q(a, b)$ shall satisfy:

$$
\begin{gathered}
\frac{\partial Q(a, b)}{\partial a}=2 \sum_{i=1}^{m}\left(a+b x_{i}-y_{i}\right)=0 \\
\frac{\partial Q(a, b)}{\partial b}=2 \sum_{i=1}^{m}\left(a+b x_{i}-y_{i}\right) x_{i}=0
\end{gathered}
$$

Equation the fitting curve satisfies is got after organization:

$$
\left\{\begin{array}{l}
m a+\left(\sum_{i=1}^{m} x_{i}\right) b=\sum_{i=1}^{m} y_{i} \\
\left(\sum_{i=1}^{m} x_{i}\right) a+\left(\sum_{i=1}^{m} x_{i}^{2}\right) b=\sum_{i=1}^{m} x_{i} y_{i}
\end{array}\right.
$$

Formula (10) is the normal equation of the fitting curve. With elimination method or Cramer method, the equation is solved:

$$
\begin{aligned}
a & =\left|\begin{array}{cc}
\sum_{i=1}^{m} y_{i} & \sum_{i=1}^{m} x_{i} \\
\sum_{i=1}^{m} x_{i} y_{i} & \sum_{i=1}^{m} x_{i}^{2}
\end{array}\right| /\left|\begin{array}{cc}
m & \sum_{i=1}^{m} x_{i} \\
\sum_{i=1}^{m} x_{i} & \sum_{i=1}^{m} x_{i}^{2}
\end{array}\right| \\
& =\left(\sum_{i=1}^{m} y_{i} \sum_{i=1}^{m} x_{i}^{2}-\sum_{i=1}^{m} x_{i} \sum_{i=1}^{m} x_{i} y_{i}\right) /\left(m \sum_{i=1}^{m} x_{i}^{2}-\left(\sum_{i=1}^{m} x_{i}\right)^{2}\right) \\
b & =\left(m \sum_{i=1}^{m} x_{i} y_{i}-\sum_{i=1}^{m} x_{i} \sum_{i=1}^{m} y_{i}\right) /\left(m \sum_{i=1}^{m} x_{i}^{2}-\left(\sum_{i=1}^{m} x_{i}\right)^{2}\right)
\end{aligned}
$$

Linear fitting by least square method employs existing sampling time points to reproduce the linear change described by these points, i.e. find a linear equation $y=a x+b$ (main point of this algorithm is how to solve factor $a$ and $b$ of the linear equation with given data).

Given data sequence $\left(x_{i}, y_{i}\right), i=1,2, \cdots, m$, fit this set of data with quadratic polynomial function.

Assume $p(x)=a_{0}+a_{1} x+a_{2} x^{2}$, work out the mean square error of the fitting function and the data sequence:

$$
Q\left(a_{0}, a_{1}, a_{2}\right)=\sum_{i=1}^{m}\left(p\left(x_{i}\right)-y_{i}\right)^{2}=\sum_{i=1}^{m}\left(a_{0}+a_{1} x_{i}+a_{2} x_{i}^{2}-y_{i}\right)^{2}
$$

According to the extremum principle of multivariate function, the minimum of $Q\left(a_{0}, a_{1}, a_{2}\right)$ satisfies.

\section{Reverse modeling of terrain}

Select surface parameters like name of surface, etc., acquire terrain data points through picking points in the layer or picture, import the terrain data points into Civil3D modeling space, and finally, click "create a surface" to finish the reverse modeling of the terrain surface. The result is as shown in Fig. 6 and Fig. 7. 


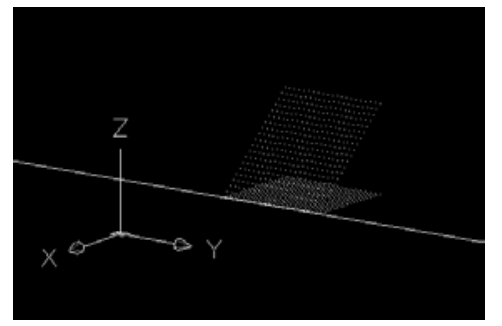

Fig.5 Generated Contour Ray

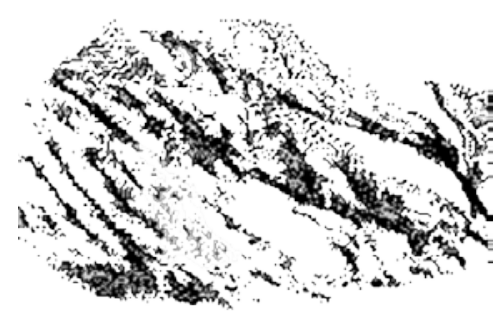

Fig.6 Point Cloud of the Terrain

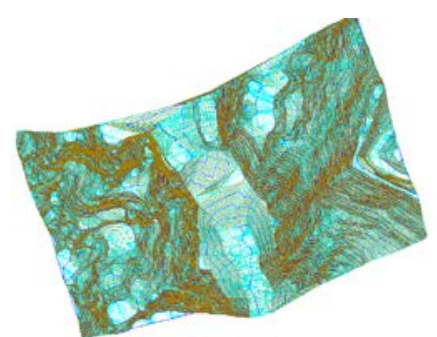

Fig.7 Terrain Surface diagram

\section{Modeling of Virtual Reality Model of an Engineering}

This project is about 4 kilometers upstream a town and is a leading reservoir with comprehensive utilization benefits. Main buildings of the hub include roller compacted concrete gravity dam, power house at dam toe, etc. The dam is of roller compacted concrete gravity type. Its crest elevation is EL.660.34m, its crest length is $736.0 \mathrm{~m}$ and its maximum dam height is $120.34 \mathrm{~m}$.

For this scanning, 21 stations are set, 7 inside the plant and 14 outside the plant, to scan the whole dam, the machine set inside the plant and the buildings in the surrounding of the dam.

After acquiring huge amounts of original point cloud data through VZ-1000 non-contact 3D laser scanner. Pre-processing of the data is finished by the cloud data processing software Riscan Pro matching with the VZ-1000 3D laser scanner.

Import the point cloud data organized in dxf format in the first station into Civil3D software, the 3D point cloud model can be divided into left bank water retaining dam section, plant dam section, flush weir section and right bank water retaining dam section for modeling according to different functions of the dam and different layers can be established correspondingly; different colors represent different layers, and the effect is as shown in Fig.8.

For quick and convenient modeling, divide the cloud data into left bank water retaining dam section, flush weir section, plant dam section and right bank water retaining dam section according to different functions of the dam, model respectively and finally splice into a whole (Fig. 8); establish 3D entity based on the 3D point cloud model as shown in Fig. 9 through Boolean operation of the 3D entity model.

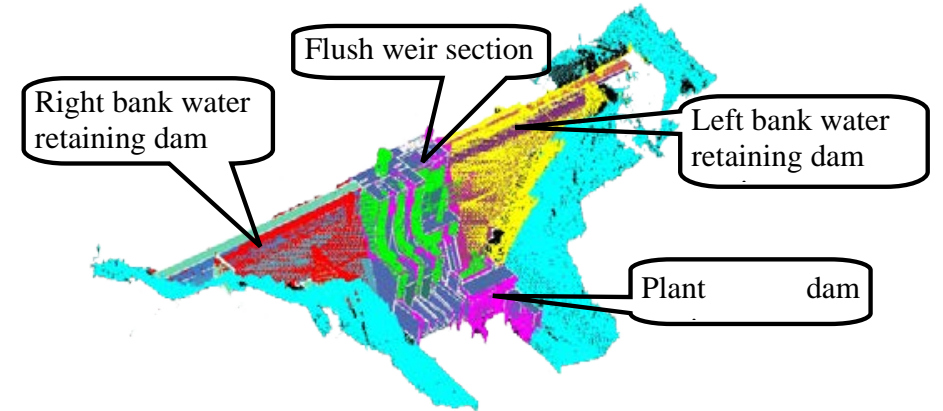

Fig. 8 Point cloud data after layering
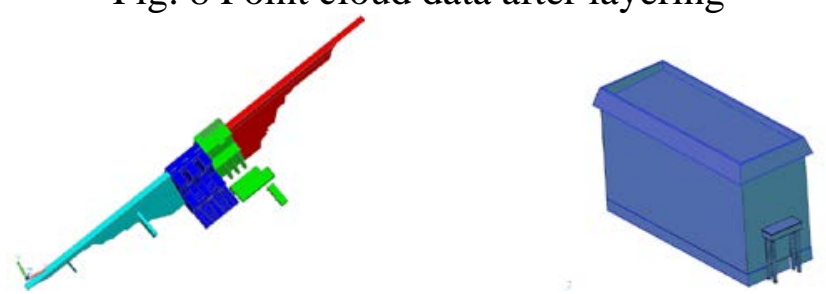

Fig. 9 3D reverse entity model of the whole dam and the plant

\section{Conclusion}

Through a systematic research on the application of methods for acquiring point cloud data concerning aerial surveying and laser scanning of modern water conservancy projects, tools are developed for processing of point cloud data of modern water conservancy projects and reverse modeling technology, with key points including fitting processing of general equation of planes, 
processing method of contour equation, fitting processing method of center line, fitting of terrain data, etc. Combined with a specific hydropower project, detailed application is carried out by step from the aspects of station distribution and acquisition of point cloud data, splicing and redundancy removal processing of data and establishment of 3D models to demonstrate that spatial information and data can be acquired rapidly through this method, and reverse 3D modeling is implemented, realizing the basic modeling for informationization of modern hydropower projects.

\section{Acknowledgement}

In this paper, the research was sponsored by Fund for Opening-up Cooperation Projects of Henan Province (142106000043), Fund for Key Science and Technology Breakthrough Projects of Henan Province (132102310037), Fund for Science and Technology Projects Jointly Taken by Chinese Academy of Sciences and Henan Province (112106000035), National Natural Science Foundation of China (No. 61471338), President Fund of UCAS, and Youth Innovation Promotion Association CAS (2015361).

\section{References}

[1]Wu Chenliang. Reverse Modeling of Buildings Based on 3D Laser Scanning Technology, 2014, (5), 9-11.

[2]Zhu Yanjuan, Zhou Laishui, Zhang Liyan. Scattered Point Cloud Data Registration Algorithm [J]. Journal of Computer-Aided Design \& Computer Graphics, 2006, 18 (4), 475-481.

[3]Luo Xianbo, Zhong Yuexian, Li Renju. Data Registration in 3-D Scanning Systems [J]. Journal of Tsinghua University (Science and Technology), 2004, 44 (8), 1104-1106.

[4]Zhang Liyan, Zhou Rurong, Cai Weibin, et al. Research on Cloud Data Simplification [J]. Journal of Computer-Aided Design \& Computer Graphics, 2001, 13 (11), 1019-1023.

[5]Liu Shangwei, Wei Lushuang. Research on the Digital Geological Multiple Source Database and 3D Modeling Method [J]. Yellow River, 2012, 34 (2), 123-125.

[6]Wei Qun. Research on and Application of Logical Product Models [C]. Collection of Thesis of New Century Water Conservancy Science and Technology Frontier (Academicians) Forum. 2005: 586-595.

[7]Wei Qun, Peng Chengshan. Establishment of Chinese and Foreign Steel Structure Material Database and Development of Application Software [C]. Proceedings of 2006 National Construction Steel Structure Assembly. 2006: 263-269.

[8]Wei Qun. Independent Research and Development of Software System for Visual Simulation 3D Entity Modeling and Virtual Reality of Digital City [C]. Proceedings of the Second China International Conference on Digital City. 2006: 204-209.

[9]Wei Qun. Research on and Application of Virtual Reality Technology and Methods for Steel Structure Engineering [C]. 2006 Academic Annual Conference on Steel Structure. 2006: 33-37.

[10]Fund for Science and Technology Projects of PowerChina. Fund for Science and Technology Projects of Guizhou Province (GZ NO.(2012)3017). 